\title{
Supplementary Material: Lipid-Protein Interactions and the Membrane/Water Interfacial Region
}

\author{
$\underline{\text { Simulation system details }}$
}

The starting structures corresponded to PDB files $1 \mathrm{k} 4 \mathrm{c}$ (for KcsA, resolution $2.0 \AA$ ) and 1bxw (for OmpA, resolution $2.5 \AA$ ). Both simulations were performed using the GROMACS MD simulation package (www.gromacs.org), and were significant extensions (by $>5 \mathrm{~ns}$ in each case) of earlier studies ${ }^{1,2}$. An extended united atom version of the GROMOS $96^{3}$ force field was used. Both proteins were inserted into preequilibrated phospholipids bilayers following the procedure described in ${ }^{4}$. Every system was energy minimized before MD, using $<1000$ steps of the steepest descent method in order to relax any steric conflict(s) generated during the set-up. Each neutral system was solvated with SPC waters (33 waters/lipid for the KcsA simulation and 46 waters/lipid for the OmpA simulation) and sufficient counter ions to make the system electically neutral. The resultant system sizes were of $\sim 50,000$ atoms. During equilibration runs, the protein was harmonically restrained with a force constant of $1000 \mathrm{kJmol}^{-1} \mathrm{~nm}^{-2}$. Longrange electrostatic interactions were calculated using the particle mesh Ewald method ${ }^{5}$. A cutoff of $10 \AA$ was used for the van der Waals interactions. Both simulations were performed at constant temperature, pressure and number of particles. The temperature of the protein, lipids, water and ions were each coupled separately, using the Berendsen thermostat ${ }^{6}$ at $300 \mathrm{~K}$ for the KcsA/POPC simulation and at $310 \mathrm{~K}$ for the OmpA/DMPC simulation, with a coupling constant $\tau_{\mathrm{T}}=0.1 \mathrm{ps}$. The pressure was coupled using the Berendsen algorithm at 1 bar with coupling constant $\tau_{P}=0.1 \mathrm{ps}$. The timestep for integration was 2 fs. The LINCS algorithm ${ }^{7}$ was used to restrain bond lengths. Further details concerning protonation states of each protein and the procedure to insert the protein in the bilayer can be found elsewhere ${ }^{1,2}$. Production runs (15 ns for each system) were performed on a 32 node Beowulf cluster. Results are presented without discarding any production run time. Analyses were performed using GROMACS tools and/or local code. In this analysis a H-bond was defined as occurring if the distance between the hydrogen and acceptor atoms was $<2.5 \AA$, and the donor-hydrogen-acceptor angle was $>$ $120^{\circ}$.

(1) Domene, C.; Sansom, M. S. P. Biophys. J. 2003, (in press), ms. 2002/018044.

(2) Bond, P.; Sansom, M. S. P. J. Mol. Biol. 2003, 329, 1035-1053.

(3) Lindahl, E.; Hess, B.; Van der Spoel, D. J. Mol. Model. 2001, 7, 306-317.

(4) Faraldo-Gómez, J.; Smith, G. R.; Sansom, M. S. P. Eur. Biophys. J. 2002, 31, 217-227.

(5) Darden, T.; York, D.; Pedersen, L. Journal of Chemical Physics 1993, 98, 1008910092.

(6) Berendsen, H. J. C.; Postma, J. P. M.; van Gunsteren, W. F.; DiNola, A.; Haak, J. R. J. Chem. Phys. 1984, 81, 3684-3690.

(7) Hess, B.; Bekker, H.; Berendsen, H. J. C.; Fraaije, J. J. Comput. Chem. 1997, 18, 1463-1472. 